\title{
IMPACTO DE UMA CAPACITAÇÃO PARA ENFERMEIROS ACERCA DA ASSISTÊNCIA NO PÓS-OPERATÓRIO DE CIRURGIA CARDÍACA
}

\author{
The impact of nurse training in cardiac surgery postoperative care
}

\section{Impacto de una capacitación para enfermeros acerca de la asistencia en el postoperatorio de cirugía cardíaca}

\author{
Marina Germani Lucas ${ }^{1 *}$, Érica Bueno Camargo de Oliveira², Isabela Cristina de Oliveira², Melissa Basseto ${ }^{3}$, Regimar Carla Machado ${ }^{5}$
}

RESUMO: Objetivo: Avaliar o impacto de um programa de capacitação teórica para enfermeiros acerca dos cuidados ao paciente no pós-operatório de cirurgia cardíaca. Método: Estudo descritivo, quantitativo, realizado em um hospital filantrópico do interior do estado de São Paulo, dividido em três etapas: aplicação do instrumento de pré-teste, capacitação expositiva dialogada e aplicação do instrumento de pós-teste. Resultados: Participaram da pesquisa 47 enfermeiros, a maioria do sexo feminino, com experiência de 1 a 3 anos na enfermagem, e somente 4 enfermeiros com experiência em cirurgia cardíaca. Verificou-se aumento significativo no número de acertos na avaliação após a capacitação, no pós-teste em relação ao pré-teste. Conclusão: Foi evidenciada a necessidade de formação continuada para os enfermeiros atuantes no pós-operatório de cirurgias cardíacas. O programa de capacitação teórica para os enfermeiros se mostrou significante, porém limitado, no que tange ao processo ensino-aprendizagem.

Palavras-chave: Período pós-operatório. Cuidados de enfermagem. Cirurgia torácica. Complicações pós-operatórias.

ABSTRACT: Objective: To evaluate the impact of a theoretical training program for nurses in care for postoperative cardiac surgery patients. Method: Descriptive, quantitative study, conducted at a philanthropic hospital in the countryside of the state of São Paulo, and divided into three stages: application of a pre-test, training with active participation, and application of a post-test. Results: A total of 47 nurses participated in the study, most of them females, with 1 to 3 years of experience in nursing, and only 4 of them had experience in cardiac surgery. We observed a significant increase in the number of right answers on the test after the training program, in comparison with the pre-test. Conclusion: The study demonstrated the need for continuing education for nurses working in postoperative of cardiac surgeries. The theoretical training program for nurses proved to be significant but limited in terms of teaching-learning process. Keywords: Postoperative period. Nursing care. Thoracic Surgery. Postoperative complications.

RESUMEN: Objetivo: Evaluar el impacto de un programa de capacitación teórica para enfermeros acerca de los cuidados al paciente en el postoperatorio de cirugía cardiaca. Método: Estudio descriptivo, cuantitativo, cumplido en un hospital filantrópico del interior del estado de São Paulo, dividido en tres etapas: aplicación del instrumento de pre-prueba, capacitación expositiva dialogada y aplicación del instrumento de post-prueba. Resultados: Participaron de la investigación 47 enfermeros, la mayoría del sexo femenino, con experiencia de 1 a 3 años en la enfermería, y solamente 4 enfermeros con experiencia en cirugía cardiaca. Se encontró un aumento significativo en el número de aciertos en la evaluación después de la capacitación, en el post-prueba en relación a la pre-prueba. Conclusión: Se ha evidenciado la necesidad de formación continuada para los enfermeros actuante en el postoperatorio de cirugías cardíacas. El programa de capacitación teórica para los enfermeros se mostró significante, pero limitado, en lo que se refiere al proceso enseñanza-aprendizaje.

Palabras clave: Periodo posoperatorio. Atención de enfermería. Cirugía torácica. Complicaciones posoperatorias.

'Graduada em Enfermagem pela Universidade Federal de São Carlos - São Carlos (SP), Brasil.

2Enfermeira mestranda do Programa de Pós-Graduação em Enfermagem da Universidade Federal de São Carlos - São Carlos (SP), Brasil.

'Enfermeira. Membro do Grupo de Estudos e Pesquisas em Saúde Baseada em Evidências e Estratégias de Ensino da Universidade Federal de São Carlos - São Carlos (SP), Brasil.

${ }^{5}$ Doutora; professora adjunto I do Departamento de Enfermagem da Universidade Federal de São Carlos - São Carlos (SP), Brasil.

*Autor correspondente: ma_germani@hotmail.com

Recebido: 21/09/2017 - Aprovado: 03/03/2018

DOl: $10.5327 / 21414-4425201800020006$ 


\section{INTRODUÇÃO}

A equipe de saúde é responsável por planejar e discutir ações que ofereçam uma assistência de qualidade, a fim de proporcionar a rápida recuperação e a alta precoce ao paciente submetido à cirurgia cardíaca. Na fase do pós-operatório, o enfermeiro, auxiliado por sua equipe, é responsável por monitorar a recuperação anestésica (RA) e atentar para possíveis complicações resultantes da cirurgia, enquanto o paciente está internado na Unidade de Terapia Intensiva (UTI $)^{1}$.

Para promover um cuidado integral, singular e de qualidade, o enfermeiro deve inserir à sua prática cotidiana o processo de enfermagem, de forma a sistematizar a assistência e adotar a prática clínica baseada em evidências². Nesse sentido, destaca-se a prescrição de cuidados como uma das fases do processo de enfermagem, a qual deve ser realizada a partir do conhecimento científico do enfermeiro e das necessidades individuais de cada paciente, analisadas mediante a coleta de dados, a elaboração dos diagnósticos de enfermagem e o planejamento de cuidados ${ }^{1}$.

Nesse contexto, é importante que os profissionais sejam treinados com frequência, com o propósito de desenvolver suas competências e habilidades para identificar as reais necessidades do paciente e planejar o seu cuidado de forma eficaz. As capacitações em diferentes temas possuem a finalidade de atualizar e complementar o conhecimento do profissional e permitir um melhor desempenho do participante, além de constituir uma necessidade da área da saúde, que perpassa por constantes mudanças e exige do enfermeiro uma qualificação profissional permanente ${ }^{3}$.

Em vista disso, a capacitação para enfermeiros atuantes na cirurgia cardíaca é de extrema importância, considerando que essa é uma das possibilidades terapêuticas adotadas no caso das doenças cardiovasculares (DCV), caracterizadas como a principal causa de óbito no mundo e registrando anualmente 17,5 milhões de casos, trazendo grandes preocupações a gestores da saúde $e^{4-6}$.

Todavia, alguns casos necessitam do tratamento cirúrgico, o qual experimenta, de forma célere e constante, notável avanço tecnológico, de forma a aumentar não só a sobrevida, mas também a qualidade de vida desses pacientes após a cirurgia.

Entre as classes de cirurgias cardíacas estão a reconstrutora, a corretora e a substitutiva. A revascularização do miocárdio (RM) é a cirurgia reconstrutora mais realizada e tem o objetivo de preservar a função do miocárdio, podendo ser realizada com ou sem circulação extracorpórea $(\mathrm{CEC})^{1}$.

A CEC é um procedimento utilizado na maioria das cirurgias cardíacas e consiste no desvio cardiopulmonar total, substituindo, temporariamente, as funções de bombeamento do coração e ventilatória dos pulmões. Os riscos de complicações, como arritmias, isquemias e alterações nos níveis pressóricos no pós-operatório, estão diretamente relacionados ao tempo em que o paciente é exposto à CEC. Quanto maior o tempo de exposição à CEC, maior a probabilidade dos pacientes apresentarem déficits neurológicos prolongados, como alteração da função cognitiva, intelectual e letargia ${ }^{2}$.

Dessa forma, pacientes submetidos à cirurgia cardiovascular, principalmente os que utilizaram a CEC, requerem cuidados sistemáticos no pós-operatório e permanecem na UTI por, em média, quatro dias, sendo que a complicação de maior frequência é a pulmonar ${ }^{1,7}$.

A ideia da aplicação do programa de treinamento ora abordado foi incitada devido à ampliação no Serviço de Cirurgia Cardiovascular de uma instituição hospitalar no interior do estado de São Paulo e, assim, atender à necessidade de capacitar enfermeiros para suprir a recente demanda.

Portanto, esta pesquisa é importante para avaliar um método de capacitação para enfermeiros que assiste o paciente no pós-operatório de cirurgia cardíaca, dado que uma assistência adequada contribui para melhoria da qualidade dos cuidados após a cirurgia.

\section{OBJETIVOS}

Avaliar o impacto de um programa de capacitação teórica para enfermeiros acerca dos cuidados ao paciente no pós-operatório de cirurgia cardíaca;

Avaliar o desempenho apresentado pelos enfermeiros antes e após o método aplicado.

\section{MÉTODO}

Trata-se de um estudo descritivo, quantitativo, de um programa de capacitação teórica acerca dos cuidados ao paciente no pós-operatório de cirurgia cardiovascular, com enfermeiros de uma instituição hospitalar do interior do estado de São Paulo. A pesquisa foi dividida em três etapas: 
1. aplicação do instrumento pré-teste;

2. capacitação teórica;

3. aplicação do instrumento pós-teste.

O instrumento de coleta de dados (pré e pós-teste) foi elaborado com base na literatura ${ }^{2,8-13}$. A primeira parte foi composta pela caracterização sociodemográfica dos participantes. A segunda parte integrou-se por 14 questões relacionadas às variáveis pertinentes à temática do estudo: cuidados no período pré, trans e pós-operatório de cirurgia cardíaca, com abordagem dos temas ventilação mecânica, monitoração hemodinâmica, cuidados com drenos, cuidados com curativos, temperatura corporal, fármacos, eletrodos de marcapasso, coleta de exames laboratoriais, realização do eletrocardiograma, cuidados na realização da radiografia de tórax e prevenção de possíveis complicações. Após sua elaboração, o instrumento foi submetido à validação de aparência.

A validação de aparência tem como objetivo avaliar se o instrumento de coleta de dados está de acordo com o que se propõe a medir ${ }^{14}$. Participaram do processo de validação cinco enfermeiras com titulação de mestres, doutoras, e com experiência em pós-operatório de cirurgia cardiovascular. A seleção das juízas foi por meio de "bola de neve ou amostragem de rede", contatadas via e-mail, com convite formal ${ }^{14}$.

As sugestões quanto à clareza do enunciado e apresentação das alternativas foram consideradas pertinentes e modificadas de acordo com orientações das juízas. As variáveis relevantes nas questões sobre tamponamento cardíaco e cuidados ao paciente com balão intra-aórtico foram incluídas no instrumento ${ }^{15,16}$.

O protocolo do estudo foi elaborado seguindo os padrões ético-legais para pesquisas envolvendo seres humanos, segundo a Resolução $n^{\circ} 466 / 2012$, do Conselho Nacional de Saúde (CNS), do Ministério da Saúde, e submetido à apreciação do Comitê de Ética em Pesquisa (CEP) da Universidade Federal de São Carlos (UFSCar), sob número CAAE 46154915.0.0000.5504.

Os participantes autorizaram a utilização dos dados dessa pesquisa, por meio da assinatura do Termo de Consentimento Livre e Esclarecido (TCLE) e as autoras garantem o anonimato dos sujeitos.

Após a elaboração do projeto, foi organizado e incluído um cronograma no sistema da instituição participante (Intranet), pelas pesquisadoras, para que todos os enfermeiros tivessem acesso.
Foram disponibilizadas quatro datas no período da tarde para realização do treinamento e os profissionais compareceram ao local de acordo com sua preferência. Cada grupo foi composto, em média, por 12 enfermeiros.

O estudo foi realizado em fevereiro de 2016 e o número de enfermeiros na instituição hospitalar era de 63 profissionais, sendo que 47 participaram do estudo. Os critérios de inclusão consistiram em: ser enfermeiro, em contrato de trabalho com a instituição sede da pesquisa, aceitar participar do estudo mediante a assinatura do TCLE e completar todas as fases da capacitação.

A etapa 1 constituiu no esclarecimento aos participantes quanto aos objetivos da pesquisa e entrega das vias do TCLE, seguido do pré-teste, com a finalidade de avaliar o domínio referente à temática pelos enfermeiros participantes.

A etapa 2 compreendeu a capacitação teórica, com duração de duas horas, em formato de aula expositiva dialogada com auxílio de texto, imagens e vídeos. Foi realizada no auditório da instituição com utilização de recursos materiais presentes no local, como: data show, tela de projeção e som. As aulas foram ministradas por duas enfermeiras com experiência em pós-operatório de cirurgia cardiovascular, pesquisadoras do estudo.

A etapa 3 constituiu a aplicação do pós-teste, com o objetivo de identificar as mudanças nas respostas após a capacitação teórica e avaliar a efetividade da capacitação. Nas etapas 1 e 3, que consistiram na aplicação dos instrumentos de coleta de dados, os participantes tiveram 45 minutos para responder as questões, tanto no pré quanto no pós-teste.

Para avaliação estatística, utilizou-se uma análise descritiva e exploratória, com o objetivo de proporcionar uma visão do comportamento geral do conjunto de dados por meio da utilização do software R. A aplicação do teste de Wilcoxon teve o objetivo de analisar a possibilidade de existir uma diferença entre a proporção de acertos do pré-teste e do pós-teste, possibilitando avaliar se houve melhora de desempenho dos profissionais após o processo de capacitação e mensurar essa diferença. O coeficiente de confiança adotado foi igual a $5 \%(\mathrm{p}<0,05)$.

\section{RESULTADOS}

Participaram do estudo 47 enfermeiros que foram avaliados segundo as características sociodemográficas (Tabela 1).

O sexo feminino foi predominante no estudo e a idade de $21(44,6 \%)$ enfermeiros estava entre 30 e 39 anos. O tempo 
de experiência profissional de 1 a 3 anos prevaleceu em $22(46,8 \%)$ sujeitos. Somente 4 enfermeiros $(8,5 \%)$ relataram possuir alguma capacitação em cirurgia cardíaca.

A Tabela 2 mostra as médias de acertos dos participantes no pré e pós-teste, relacionadas ao tempo de experiência e ao aumento das médias após a capacitação. Observa-se que, no pré-teste, participantes com 6 meses de experiência tiveram, em média, 8 acertos; com 1 a 3 anos possuíam, em média, 6,68 acertos; com 3 a 5 anos, 5,7 acertos, em média; 5 a 10 anos, média de 5,12 acertos; e participantes com mais de 10 anos de experiência possuíam uma média de 8,16 acertos. Pode-se dizer que mesmo entre os participantes com mais

Tabela 1. Caracterização sociodemográfica dos participantes da pesquisa. São Carlos, 2017.

\begin{tabular}{|l|l|l|}
\hline Variável & \multicolumn{1}{|c|}{$\mathbf{n}$} & $\%$ \\
\hline Faixa etária (anos) & \multicolumn{1}{|c|}{} \\
\hline De 20 a 29 & 21 & 38,2 \\
\hline De 30 a 39 & 05 & 18,6 \\
\hline De 40 a 49 & 03 & 6,3 \\
\hline De 50 a 59 & & \\
\hline Sexo & 40 & 85,1 \\
\hline Feminino & 07 & 14,8 \\
\hline Masculino & & \\
\hline Tempo de experiência profissional & 01 & 2,1 \\
\hline 6 meses & 22 & 46,8 \\
\hline Mais de 1 ano até 3 anos & 10 & 21,2 \\
\hline Mais de 3 anos até 5 anos & 08 & 17,0 \\
\hline Mais de 5 anos até 10 anos & 06 & 12,7 \\
\hline Mais de 10 anos & 47 & 100,0 \\
\hline Experiência profissional em cirurgia cardíaca & \\
\hline Sim & 04 & 8,5 \\
\hline Não & 43 & 91,5 \\
\hline Total & & \\
\hline
\end{tabular}

de 10 anos de experiência, tendo a maior média, o tempo de experiência não influenciou na média de acertos, pois não foi observado um aumento gradativo das médias junto ao aumento do tempo de experiência.

É possível verificar que todas as médias de acertos em relação ao tempo de experiência na Enfermagem aumentaram após o processo de capacitação dos profissionais (Tabela 2), sendo que o maior aumento foi entre os participantes com 5 a 10 anos de experiência (2,75 de acertos) e o menor aumento foi entre os participantes com mais de 10 anos de experiência (0,167 de acerto). A categoria com seis meses de experiência não é conclusiva, pois contém apenas uma observação.

$\mathrm{Na}$ Tabela 3, observa-se que a variável relacionada a complicações graves no pós-operatório foi a que obteve maior número de acertos entre os participantes no pré e pós-teste. A questão que obteve menos acertos foi correspondente ao reconhecimento do tamponamento cardíaco (questão 14), totalizando somente quatro acertos no préteste e nenhum acerto no pós-teste.

Na maioria das questões do pós-teste houve aumento na quantidade de acertos em relação ao pré-teste. Houve exceção da questão 9, a respeito do tema pré-carga, com 14 acertos, e da questão 14 , sobre o reconhecimento do tamponamento cardíaco, com nenhum acerto no pósteste. A questão 1, referente às intervenções educativas, teve um acerto a menos no pós-teste, quando comparado ao pré-teste. O maior número de acertos após a capacitação ocorreu na questão 13 , referente às complicações graves no pós-operatório, com 41 acertos. Nas questões 4, 6, 8 e 10 houve aumento de mais de $20 \%$ no número de acertos após a capacitação dos enfermeiros (Tabela 3).

É possível observar que, em geral, a quantidade de acertos aumentou após o processo de capacitação dos profissionais e obteve nível de significância $(\mathrm{p}=0,0037)$. Por meio da análise estatística realizada, pode-se concluir que o desempenho dos profissionais melhorou após a capacitação teórica, como demonstram os resultados do pós-teste.

Tabela 2. Média de acertos dos participantes no pré e pós-teste relacionado ao tempo de experiência. São Carlos, 2017.

\begin{tabular}{|l|c|c|c|c|}
\hline Tempo de experiência profissional & Quantidade & $\begin{array}{c}\text { Média } \\
\text { pré-teste }\end{array}$ & $\begin{array}{c}\text { Média } \\
\text { pós-teste }\end{array}$ & $\begin{array}{c}\text { Aumento } \\
\text { após o teste }\end{array}$ \\
\hline 6 meses & 01 & 8,0 & 9,0 & 1,0 \\
\hline 1 a 3 anos & 22 & 6,681 & 7,27 & 0,589 \\
\hline 3 a 5 anos & 10 & 5,7 & 7,2 & 7,5 \\
\hline 5 a 10 anos & 08 & 5,125 & 7,875 & 2,75 \\
\hline Mais de 10 anos & 06 & 8,166 & 8,333 & 0,167 \\
\hline
\end{tabular}




\section{DISCUSSÃO}

Os profissionais de enfermagem devem estar constantemente atualizados, visto que a área da saúde passa por progressivas transformações mediante novas descobertas científicas e tecnológicas. Diante dessa situação, é imprescindível que o profissional esteja em permanente aperfeiçoamento, a fim de que o cuidado seja de qualidade, com foco na sistematização e na integralidade ${ }^{3}$.

Nessa lógica, é importante que o enfermeiro seja um dos principais agentes de transformação no trabalho em saúde e desenvolva tanto as habilidades para o cuidado, gestão e liderança, quanto para a educação, priorizando a sua própria formação continuada e a de sua equipe ${ }^{3}$.

O estudo avaliou um programa de capacitação teórica para 47 enfermeiros acerca dos cuidados ao paciente no pós-operatório de cirurgia cardíaca, sendo desenvolvido em uma instituição hospitalar filantrópica que realiza grande número de cirurgias cardiovasculares em pacientes adultos, tais como as ressecções de tumores, correções de aneurismas, dissecções aórticas, revascularização do miocárdio e troca valvar.

Contudo, foi possível evidenciar, nos resultados deste estudo, que apenas $4(8,5 \%)$ enfermeiros tinham experiência em cuidados no pós-operatório de cirurgia cardíaca, corroborando outros estudos em que os profissionais atuantes no cuidado de pacientes críticos apresentam formação profissional recente, sem experiência ou em busca de aperfeiçoamento teórico. Todavia, são pontos necessários para atuar nessa área complexa, a qual exige o desenvolvimento de habilidades ${ }^{17,18}$.

O perfil desses enfermeiros normalmente volta-se para profissionais mais responsáveis e com iniciativa, para que se adequem ao perfil de intensivista, buscando sempre atualização e aperfeiçoamento do conhecimento em cursos de especialização, para que o profissional se torne mais competente para a atuação no cuidado ao paciente crítico ${ }^{17,18}$.

Assim, como estratégia de capacitação, algumas instituições realizam treinamentos na fase de integração do profissional de Enfermagem, com o objetivo de aprimorar o cuidado ao paciente assistido. No entanto, essa prática não é normatizada por todas as instituições. Além disso, funcionários sem experiência prévia são frequentemente transferidos para setores de maior complexidade. Por conseguinte, a atualização dos profissionais deve ser estimulada periodicamente para reforço do conhecimento adquirido ${ }^{3}$.

Após a exposição teórica, os enfermeiros apresentaram melhor desempenho, sendo as questões nas quais houve maiores diferenças, as relacionadas a: balão intra-aórtico, revascularização do miocárdio e administração de adrenalina. O tempo reduzido de aplicação do segundo teste pode ter influenciado esse resultado, uma vez que a aula teórica havia sido aplicada no mesmo dia.

Um dos métodos mais utilizados para a capacitação de enfermeiros é a aula expositiva dialogada. Por meio desse

Tabela 3. Número de acertos apresentados pelos enfermeiros acerca dos cuidados ao paciente no pós-operatório de cirurgia cardíaca, no pré e no pós-teste. São Carlos, 2017.

\begin{tabular}{|l|c|c|c|c|}
\hline \multirow{2}{*}{ Variável } & \multicolumn{2}{|c|}{ Pré-teste } & \multicolumn{3}{c|}{ Pós-teste } \\
\cline { 2 - 5 } & $\mathbf{n}$ & $\mathbf{9}$ & $\mathbf{n}$ & $\%$ \\
\hline 1. Intervenções educativas no pré-operatório & 38 & 80,8 & 37 & 78,7 \\
\hline 2. Cuidados no pré-operatório & 30 & 63,8 & 32 & 68,0 \\
\hline 3. Substituição valva mitral & 16 & 34,0 & 18 & 38,2 \\
\hline 4. Revascularização do miocárdio & 06 & 12,7 & 21 & 44,6 \\
\hline 5. Equipamentos e materiais no pós-operatório & 31 & 65,9 & 35 & 74,4 \\
\hline 6. Exames no pós-operatório & 25 & 53,1 & 39 & 82,9 \\
\hline 7. Intervenções prioritárias no pós-operatório & 36 & 76,5 & 37 & 78,7 \\
\hline 8. Débito cardíaco & 21 & 44,6 & 31 & 65,9 \\
\hline 9. Pré-carga & 24 & 51,0 & 14 & 29,7 \\
\hline 10. Administração de noradrenalina & 05 & 10,6 & 17 & 36,1 \\
\hline 11. Balão intra-aórtico & 05 & 10,6 & 11 & 23,4 \\
\hline 12. Consequência do aumento do débito cardíaco & 21 & 44,6 & 25 & 53,1 \\
\hline 13. Complicações graves no pós-operatório & 40 & 85,1 & 41 & 87,2 \\
\hline 14. Reconhecimento do tamponamento cardíaco & 04 & 8,5 & 0 & 0 \\
\hline
\end{tabular}


método, o facilitador realiza a exposição de um tema e, concomitantemente, proporciona aos profissionais a oportunidade de expor vivências prévias e de aprimorar o saber, a fim de melhorar a qualidade da assistência prestada aos pacientes ${ }^{19}$.

Não obstante, o reduzido espaço de tempo para aprofundamento das reflexões, a transmissão de ideias no sentido vertical (professor/aluno) e o processo avaliativo focado na reprodução do conteúdo exposto são algumas limitações desse tipo de método, quando aplicados isoladamente ${ }^{18}$. No entanto, mesmo com essas limitações, o teste de significância foi favorável ( $\mathrm{p}=0,0037)$ no presente estudo.

Apesar dos resultados obtidos apresentarem um nível de significância satisfatório, as repercussões desta pesquisa implicam novos questionamentos para o desenvolvimento do conhecimento e de habilidades no processo de trabalho em saúde. Destarte, indagando se estratégias de ensino-aprendizagem com metodologias ativas podem atingir resultados melhores quanto ao processo de formação continuada em cuidados pós-operatórios de cirurgia cardíaca, contrapondo o método expositivo para suprir essa demanda.

A questão 14, "reconhecimento do tamponamento cardíaco", que está relacionada a uma das complicações no pós-operatório de cirurgia cardiovascular, apresentou diminuição do índice de acertos, mesmo após a capacitação, o que talvez possa ser justificado pelo método teórico oferecido, visto que há dificuldade para reflexão oferecida.

Baseado nessa indagação, é indispensável salientar a metodologia ativa como uma grande tendência dos processos educativos, uma vez que esse método possui características como autonomia do indivíduo, aprendizagem com significado e problematização para a busca de soluções, implicando em um ato de ação-reflexão-ação, capaz de gerar processos de continuidade ou de ruptura. No processo de continuidade, o sujeito confronta os conceitos apreendidos aos conhecimentos adquiridos anteriormente, ao passo que, na ruptura, o educando transcende suas vivências por meio de oportunidades de novos desafios ${ }^{20}$.

Diante desse contexto, destacam-se como limitações do estudo, as questões 9, "pré-carga", e 14, "tamponamento cardíaco", que apresentaram diminuição na quantidade de acertos no pós-teste. Esse resultado indica que houve entrave no método aplicado para a capacitação referente a esses assuntos, que estão diretamente relacionados às complicações no pós-operatório de cirurgia cardiovascular. Sobretudo, cabe enfatizar a necessidade de aplicar estratégias ativas que proporcionem melhor domínio da temática.

\section{CONCLUSÃO}

A necessidade de formação continuada para os enfermeiros atuantes na área de cuidados pós-operatórios de cirurgias cardíacas foi evidenciada neste estudo. O programa de capacitação teórica para os enfermeiros demonstrou-se significante, havendo aumento significativo do número de acertos no pósteste, em relação ao pré-teste $(\mathrm{p}=0,0037)$, embora limitado no que tange ao processo ensino-aprendizagem.

Sugere-se a realização de novos estudos para a avaliação de estratégias de capacitação para profissionais da saúde, destacando a dificuldade de encontrar estudos na literatura que envolvam treinamentos associados ao cuidado com o paciente em pós-operatório de cirurgia cardiovascular.

\section{REFERÊNCIAS}

1. Barretta JC, Auda JM, Antoniolli D, Barancelli MDC. Pós-operatório em cirurgia cardíaca: refletindo sobre o cuidado de enfermagem. Rev Fund Care [Internet]. 2017 [citado 12 set. 2017];9(1):259-64. Disponível em: http://www.seer.unirio.br/index.php/cuidadofundamental/article/ view/4042/pdf. http://dx.doi.org/10.9789/2175-5361.2017.v9i1.259-264

2. Oliveira JMA, Silva AMF, Cardoso SB, Lima FF, Zierer MS, Carvalho ML. Complicações no pós-operatório de cirurgia cardiovascular com circulação extracorpórea. Rev Interd [Internet]. 2015 [citado 12 set. 2017];8(1):9-15. Disponível em: http://revistainterdisciplinar. uninovafapi.edu.br/index.php/revinter/article/view/584/pdf_177

3. Simões TR, Vannuchi MTO, Rossaneis MA, Silva LG, Haddad MCL, Jenal S. Educação continuada: concepção de enfermeiros em hospital filantrópico de alta complexidade. Rev Enferm [Internet]. 2013 [citado 12 set. 2017];21(5):642-7. Disponível em: http://www.e-publicacoes. uerj.br/index.php/enfermagemuerj/article/view/10042/8069

4. Stipp MAC, Souza AA, Santos RS. Cardiovascular diseases and their risk factors - an analysis on the theme. Online Braz J Nurs. 2008;7. http://dx.doi.org/10.5935/1676-4285.20081229

5. Dutra D, Duarte M, Albuquerque K, Lima A, Santos J, Souto H. Cardiovascular disease and associated factors in adults and elderly registered in a basic health unit. J Res Fundam Care [Internet]. 2016 [citado 12 set. 2017];8(2):4501-9. Disponível em: http://seer.unirio. $\mathrm{br} /$ index.php/cuidadofundamental/article/view/4787/pdf_1905. DOI: 10.9789/2175-5361.2016.v8i2.4501-4509 
6. Brasil. Cerca de 17,5 milhões pessoas morrem de doenças cardiovasculares todos os anos [Internet]. Brasília: Portal Brasil; 2016 [citado 12 set. 2017]. Disponível em: http://www.brasil.gov. br/saude/2016/09/cerca-de-17-5-milhoes-pessoas-morrem-dedoencas-cardiovasculares-todos-os-anos

7. Cani KC, Araujo CLP, Karloh M, Alexandrino DFH, Palú M, Rojas DB, et al. Características clínicas de pacientes submetidos à cirurgia de revascularização do miocárdio. ASSOBRAFIR Ciência [Internet]. 2015 Dez [citado 15 set. 2017];6(3):43-54. Disponível em: http://www.uel. $\mathrm{br} /$ revistas/uel/index.php/rebrafis/article/view/21754/17846

8. Umann J, Guido LA, Linen GFC, Freitas EO. Enfermagem perioperatória em cirurgia cardíaca: revisão integrativa da literatura. Rev Min Enferm [Internet]. 2011 [citado 13 nov. 2015];15(2):275-81. Disponível em: http://www.reme.org.br/artigo/detalhes/36. http://www.dx.doi.org/ S1415-27622011000200017

9. Lira ALBC, Araújo WM, Souza NTC, Frazão CMFQ, Medeiros ABA. Mapeamento dos cuidados de enfermagem para pacientes em pósoperatório de cirurgia cardíaca. Rev Rene [Internet]. 2012 [citado 13 nov. 2015];13(5):1171-81. Disponível em: http://repositorio.ufc.br/ ri/bitstream/riufc/4587/1/2012_art_albclira.pdf

10. Duarte SCM, Stipp MAC, Mesquita MGR, Silva MM. Enfermagem no pós-operatório de cirurgia cardíaca. Esc Anna Nery [Internet]. 2012 [citado 13 nov. 2015];16(4):657-65. Disponível em: http://www. redalyc.org/pdf/1277/127728365003.pdf

11. Liakopoulos OJ, Choi YH, Haldenwang PL, Strauch J, Wittwer T, Dörge $\mathrm{H}$, et al. Impact of preoperative statin therapy on adverse postoperative outcomes in patients undergoing cardiac surgery: a meta-analysis of over 30,000 patients. Eur Heart J [internet]. 2008 [citado 13 nov. 2015];29(12):1548-59. Disponível em: https://academic.oup.com/ eurheartj/article-lookup/doi/10.1093/eurheartj/ehn198. https://doi. org/10.1093/eurheartj/ehn198

12. Soares GMT, Ferreira DCS, Gonçalves MPC, Alves TGS, David FL, Henriques KMC, et al. Prevalência das principais complicações pós-operatórias em cirurgias cardíacas. Rev Bras Cardiol [Internet]. 2011 [citado 13 nov. 2015];24(3):139-46. Disponível em: http://www. onlineijcs.com/detalhes/225/prevalencia-das-principais-complicacoespos-operatorias-em-cirurgias-cardiacas---prevalence-of-majorpostoperative-complications-in-cardiac-surgery
13. Virani SS, Nambi V, Razavi M, Lee VV, Elayda M, Wilson JM, et al. Preoperative statin therapy is not associated with a decrease in the incidence of postoperative atrial fibrillation in patients undergoing cardiac surgery. Am Heart J [Internet]. 2008 [citado 13 nov. 2015];155(3):541-6. http://dx.doi.org/10.1016/j.ahj.2007.10.027

14. Polit DF, Beck CT, Hungler BP. Fundamentos de pesquisa em enfermagem: métodos, avaliação e utilização. Porto Alegre: Artmed; 2011. p.414-5.

15. Galdeano LE, Rossi LA. Construção e validação de instrumentos de coleta de dados para o período perioperatório de cirurgia cardíaca. Rev Latino-am Enferm [Internet]. 2002 [citado 13 nov. 2015];10(6):800-4. Disponível em: http://www.revistas.usp.br/rlae/ article/view/1720/1765

16. Bellucci Júnior JA, Matsuda LM. Construção e validação de instrumento para avaliação do acolhimento com classificação de risco. Rev Bras Enferm [Internet]. 2012 [citado 17 set. 2017];65(5):751-7. Disponível em: http://www.scielo.br/scielo. php?script=sci_arttext\&pid=S0034-71672012000500006

17. Santos FC, Camelo SH. O enfermeiro que atua em unidades de terapia intensiva: perfil e capacitação profissional. Cultura cuidados [Internet]. 2015 [citado 16 set. 2017];19(43). Disponível em: https:// rua.ua.es/dspace/bitstream/10045/52599/1/Cult_Cuid_43_13.pdf

18. Santos APA, Camelo SHH, Santos FC, Leal LA, Silva BR. Nurses in post-operative heart surgery: professional competencies and organization strategies. Rev Esc Enferm USP [Internet]. 2016 [citado 16 set. 2017];50(3):472-8. http://dx.doi.org/10.1590/ S0080-623420160000400014

19. Miotto HC, Camargos FRS, Ribeiro CV, Goulart EMA, Moreira MCV. Efeito na RCP utilizando treinamento teórico versus treinamento teórico-prático. Arq Bras Cardiol [Internet]. 2010 [citado 16 set. 2017];95(3). Disponível em: http://www.scielo.br/scielo. php?script=sci_arttext\&pid=S0066-782X2010001300008

20. Gonçalves MFC, dos Santos R, Silva MA, Fortuna CM, Andrade LS. Experience-based learning in nursing teacher education: a historicalcultural research study. Am J Educ Research [Internet]. 2014 [citado 16 set. 2017];2(5):316-24. Disponível em: http://pubs.sciepub.com/ education/2/5/12/ 